Published in final edited form as:

Clin Lymphoma Myeloma Leuk. 2013 February ; 13(1): 48-54. doi:10.1016/j.clml.2012.07.009.

\title{
Current Practices in the Management of Chronic Myeloid Leukemia
}

\author{
Hagop M. Kantarjian ${ }^{1}$, Richard A. Larson ${ }^{2}$, Jorge E. Cortes ${ }^{1}$, Kathleen L. Deering ${ }^{3}$, and \\ Michael J. Mauro 4 \\ ${ }^{1}$ Department of Leukemia, The University of Texas M.D. Anderson Cancer Center, Houston, TX \\ ${ }^{2}$ Department of Medicine, University of Chicago, Chicago, IL \\ ${ }^{3} \mathrm{EPI}-\mathrm{Q}$, Inc, Oak Brook, IL \\ ${ }^{4}$ Center for Hematologic Malignancies, Knight Cancer Institute at Oregon Health \& Science \\ University, Portland, OR
}

\begin{abstract}
A cross-sectional survey of board certified hematologists/oncologists was conducted to describe current chronic myeloid leukemia (CML) practice patterns and compare these self-reported practices with the clinical guidelines. Overall, the reported practice patterns regarding CML treatment were in accordance with guidelines; however, decisions also appear to be based on the attitudes, beliefs, and personal experience of the responding physicians.
\end{abstract}

Background-A previous survey of physician self-reported practice patterns in the management of CML was conducted in 2005. The National Comprehensive Cancer Network and European LeukemiaNet guidelines now include nilotinib and dasatinib in their treatment algorithms for CML. To assess these new guidelines, a cross-sectional survey of US hematologists and/or oncologists was conducted in December 2010 through an online survey.

Materials and Methods-The survey had 43 questions consisting of items updated from the 2005 survey to reflect changes in clinical practice, tyrosine kinase inhibitor therapy, and current guidelines.

Results-Analysis of the responses from 507 board certified medical oncologists/hematologists suggests that the use of imatinib $400 \mathrm{mg}$ as an initial treatment option had decreased from $62 \%$ in 2005 to $52 \%$ in the 2010 survey. Currently, nearly $40 \%$ of physicians would choose either nilotinib or dasatinib as first-line treatment. From the surveyed physicians, achievement of at least a major molecular response (MMR) is the predominant treatment goal in chronic phase CML.

Published by Elsevier Inc.

Address for correspondence: Hagop M. Kantarjian, MD, The University of Texas, M.D. Anderson Cancer Center, 1515 Holcombe Blvd, Houston, TX 77030 Fax: 713-792-2031; hkantarj@mdanderson.org.

Presented in part at the American Society of Clinical Oncology Annual Meeting in Chicago, IL, June 3-7, 2011, and the American College of Clinical Pharmacy Annual Meeting in Pittsburgh, PA, October 16-19, 2011.

Supplementary Data

Supplementary material from this article is available online at cigjournals.com.

Disclosures

Dr Kantarjian has received research funding from Novartis, Bristol-Myers Squibb, and ARIAD, and has consulted for Novartis; Dr Larson has consulted or advised Novartis; Dr Cortes has participated in advisory boards for Bristol-Myers Squibb, ARIAD, and Chem-Genex; Dr Deering is an employee of EPI-Q, Inc, an independent health outcome and economics research group contracted by No-vartis Pharmaceutical Corporation; and Dr Mauro has served as an adviser and consultant for BMS and Novartis. 
Conclusion-This survey emphasizes the need for continued updates and education regarding optimal therapy, monitoring practices, and therapeutic end points in CML.

\section{Keywords}

CML; Guidelines; Practice patterns; Survey; Tyrosine kinase inhibitors

\section{Introduction}

A survey was conducted between November 2005 and January 2006 to assess hematologists' and oncologists' self-reported treatment strategies for chronic myeloid leukemia (CML). ${ }^{1}$ The results suggested that practice patterns of respondents were generally aligned with current guidelines and published clinical trials. However, there were some notable findings, including dosing discrepancies of tyrosine kinase inhibitors (TKI), confusion on the optimal timing of treatment decisions, and time points for evaluating therapeutic response. The survey results also suggested that the use of molecular monitoring was less than optimal, and a full appreciation of the differences between the qualitative and quantitative polymerase chain reaction (PCR) techniques was missing despite developments in real-time, reproducible, quantitative assays. The authors concluded that there were several areas where the practice of treating CML could be improved. Additionally, a recommendation was made to repeat the survey in the future to identify changing trends in treatment patterns.

In the 5 years since the original survey, practice has changed with respect to disease monitoring in CML and in the choice of first-line treatment options. In addition to the previously established standard of care, imatinib, the FDA granted approval for the use of nilotinib $^{2}$ (Tasigna; Novartis Pharmaceuticals Corp, East Hanover, NJ) and dasatinib ${ }^{3}$ (Sprycel; Bristol-Myers Squibb Co, Princeton, NJ) based on the results of clinical studies supporting the use of these agents in the initial treatment of CML. Subsequently, the National Comprehensive Cancer Network (NCCN; 2010) ${ }^{4}$ and European LeukemiaNet $(E L N ; 2009)^{5}$ guideline recommendations were amended to include nilotinib and dasatinib into their treatment algorithms for newly diagnosed CML. In concert with these changes, the ELN guidelines ${ }^{5}$ updated their definitions for imatinib response (optimal, failure, suboptimal) and warning prognostic factors in patients with chronic phase CML.

The purpose of this study was to re-evaluate self-reported management of CML among hematologists and/or oncologists within the United States given multiple changes in CML treatment options and response expectations in the past 5 years. This survey evaluates physicians' perceptions regarding current CML therapies, treatment associated issues such as toxicity and resistance, efficacy, ease of use of available treatments, barriers to optimal treatment, monitoring, and determination of treatment effectiveness. The results of this survey are discussed in relation to the original survey, current guidelines, and recent clinical trial outcomes.

\section{Materials and Methods}

This was a prospective United States-based, noninterventional, cross-sectional study conducted through an online survey in December 2010. The 43-question survey was expected to be completed in 20 minutes and consisted of items updated from the previous survey ${ }^{1}$ to reflect new clinical evidence, change in clinical practice, and updated clinical guidelines. The survey was reviewed and approved by the investigators for content and clarity of the questions and instructions, and was pilot tested by Medefield, the survey 
company, to determine the length and feasibility of the survey. The survey questions and responses are available in the Supplementary Data.

The survey population included physicians who have registered with the survey company and had agreed to be contacted for the purposes of survey research. The survey company verified the credentials of physicians opting in for survey research through US medical education numbers and educational history (ie, diplomas). Physicians were eligible if they reported being a board certified hematologist, oncologist, or hematologist/oncologist; and treated at least 5 patients with CML outside of the context of a clinical trial in the past 2 years.

Five hundred seven physicians treating patients with CML were surveyed. The surveyed physicians represented the first 507 that qualified, from a population of US physicians who were registered with the survey company and who fit the criteria established by the survey (convenience sample).

The variables collected were descriptive in nature. As such, the frequency and percent of each response category was calculated. In addition, responses were examined by physician practice type.

\section{Results}

\section{Survey Participant Characteristics}

Eighty-two percent of the respondents specialized in hematology/oncology and the remaining $18 \%$ were in the field of medical oncology. The majority were in private practice $(57.4 \%)$ followed by employment within a university or teaching institution (26.4\%) or community or regional hospitals (14.6\%). For more than $90 \%$ of respondents, it had been more than 6 years since completing their medical training and $89 \%$ had CML patients under their direct management at the time of the survey. Half of the respondents had patients enrolled in clinical trials (53.7\%) ranging from 1 to more than than 20.

\section{Initial Diagnosis and Workup}

Participants were asked to respond to questions referring to diagnostic tests typically performed at initial patient workup. With multiple answers allowed, peripheral blood cell counts $(84.6 \%)$ and bone marrow cytogenetics $(83.4 \%)$ were cited as the most commonly obtained diagnostic tests when evaluating a patient with a consistent picture of CML (question [q.] 7). This was followed by bone marrow fluorescence in situ hybridization (FISH) (63.7\%), peripheral blood FISH (59\%), and quantitative reverse-transcriptase PCR (qRT-PCR) (57.2\%).

For purposes of diagnosis, participants most frequently relied on bone marrow cytogenetics (65.5\%), followed by bone marrow and peripheral blood FISH (each 48.1\%), and peripheral blood cell counts (44.4\%) (q. 8). qRT-PCR was included less often for purposes of diagnosis (36.7\% peripheral blood qRT-PCR, $24.7 \%$ bone marrow qRT-PCR). When asked the frequency of including a bone marrow study at the initial diagnosis, $80 \%$ of survey respondents indicated that it is performed $>90 \%$ of the time (q. 9).

\section{Treatment Patterns by Patient Type}

The survey contained several clinical scenarios for which the respondents were to choose their preferred treatment option. Two of the cases involved a newly diagnosed 40-year-old patient with chronic phase CML; 1 had an human leukocyte antigen-matched related sibling (q. 10) and the other an unrelated matched donor (q. 11). The third case was a newly 
diagnosed 60-year-old patient who was at high risk for transplantation complications because of comorbidities (q. 12). Approximately $60 \%$ of the respondents preferred to use imatinib and $40 \%$ preferred to use nilotinib/dasatinib as initial therapy in each of the cases. In the case with a matched related sibling, the opinion was split evenly as to whether an allogeneic stem cell transplant (allo-SCT) evaluation should be performed in addition to imatinib/nilotinib/dasatinib (q. 10). However, with an unrelated donor, more than $60 \%$ would not recommend allo-SCT evaluation at the onset of therapy (q. 11).

\section{Treatment Goals and Monitoring}

Seventy-two percent of respondents indicated that their primary goal for treatment response was major molecular response (MMR; 27.4\%) or complete molecular response (CMR; 44.4\%) for a newly diagnosed CML patient with TKI therapy (q. 13). Seventeen percent consider the achievement of complete cytogenetic response (CCyR) to be their primary goal in treating CML patients (Figure 1). Based on the respondents' primary treatment goal, the survey then queried the most critical time period to evaluate initial response to TKI therapy and the results are displayed in Figure 2 (q. 14).

Figure 3 illustrates when respondents indicated 'effective' initial treatment for newly diagnosed CML patients receiving TKI therapy among hematologic remission (HR), partial cytogenetic response (PCyR), CCyR, and MMR (q. 25).

In addition to peripheral blood counts, the majority of respondents assessed the initial response to TKI therapy by choosing a combination of tests (q. 15). Allowing for multiple answers, the most common tests were qRT-PCR (66.3\%), peripheral FISH (46.7\%), and bone marrow cytogenetics $(28.8 \%)$. The combinations of responses given to monitor initial response to TKI therapy are displayed in Figure 4. Additionally, respondents indicated the first bone marrow examination for cytogenetic response after initiation of TKI therapy should occur at 6 months or later (40.6\% at 6 months, $24.5 \%$ at 12 months) (q. 16).

The frequency of continued cytogenetic analysis for monitoring TKI therapy in patients with documented CCyR was reported to occur at 6 month intervals (37.1\%) or longer (33.7\%) (q. 18). For patients not achieving CCyR, cytogenetic monitoring was reported to be more frequent with $53.1 \%$ suggesting to test every 3 months or less (q. 19). When patients taking TKI therapy achieve CCyR, monitoring with qRT-PCR for breakpoint cluster regionabelson (BCR-ABL) was reported to routinely be performed every 3 months (47\%) and every 6 months (37.5\%) (q. 20). Five percent of respondents would not routinely use qRTPCR after CCyR; $2 \%$ indicated they would routinely monitor every 1 to 2 months.

In a case-based question involving a 70-year-old woman with CML who recently started taking imatinib $400 \mathrm{mg}$ orally daily, respondents were asked to choose the test or multiple tests they used for monitoring (q. 23). The frequencies were 79.3\% peripheral blood counts; $31.6 \%$ cytogenetics; $41.8 \%$ FISH; and $49.1 \%$ qRT-PCR. The combination strategies reported by $10 \%$ or more for monitoring initial response to therapy were $21.9 \%$ peripheral blood counts alone; $12.4 \%$ peripheral blood count and qRT-PCR; $11.2 \%$ peripheral blood counts, cytogenetics, FISH, and qRT-PCR; $10.3 \%$ peripheral blood counts and FISH; and $10.1 \%$ qRT-PCR alone.

\section{Suboptimal Response to Therapy}

Table 1 displays the survey results for determination of suboptimal response in both general and case-based question formats. The general question related to suboptimal response to any initial TKI therapy (q. 33) and the case-based question was specific to imatinib suboptimal response (q. 24). The response options for these questions were adapted from the ELN definitions $s^{5}$ for suboptimal response and/or treatment failure with first-line imatinib; 
therefore, all were plausible options for determining if suboptimal response or treatment failure was evident so respondents were asked to choose all answers that applied. In patients achieving CCyR but not MMR after 2 years on imatinib $400 \mathrm{mg}$ daily, respondents would most frequently recommend increasing the imatinib dose (51.9\%) or changing to a nilotinib/ dasatinib regimen (34.3\%) (q. 34, multiple answers allowed).

In addition to peripheral blood counts, practitioners use a variety of tests in patients with suspected treatment resistance (q. 17). The tests frequencies reported were 64.3\% qRT-PCR, $58 \%$ BCR-ABL kinase domain mutations, $40.6 \%$ bone marrow cytogenetics, $30.2 \%$ bone marrow FISH, and 29.6\% peripheral blood FISH.

\section{Treatment Failure Case-Based Questions}

A case-based question described a 65-year-old patient with CML in whom imatinib $400 \mathrm{mg}$ daily had failed (using the respondent's standard criteria) who had a matched related donor, $69 \%$ of respondents would change therapy to nilotinib or dasatinib versus $19 \%$ would change to imatinib $800 \mathrm{mg}$ (q. 40). In the same case without a matched related donor, 73\% of respondents would change therapy to nilotinib or dasatinib with $18 \%$ increasing the imatinib dose to $800 \mathrm{mg}$ (q. 41).

For a 50-year-old patient on first-line TKI therapy with a cytogenetic relapse 18 months after initially achieving CCyR and a matched related sibling, $45 \%$ would change to an alternative TKI and 36\% would change to an alternative TKI and prepare for an allo-SCT (q. 43).

Finally, assessing a 50-year-old patient previously taking imatinib and then switched to a second-line TKI because of suboptimal response or treatment failure with a matched related sibling, 50\% would treat with either nilotinib or dasatinib and $40 \%$ would perform an immediate allo-SCT if the physician found that there was a loss in cytogenetic or molecular response (q. 42).

\section{Treatment Adverse Effects and Tolerability}

Most of the physicians surveyed (85.6\%) reported using frequent physician visits early in the course of therapy as a method to monitor for treatment-related adverse events (q. 26). Toxicities reported by at least $50 \%$ of physicians included: fluid retention (70.6\%); nausea/ vomiting (68.2\%); diarrhea (68.2\%); grade 1-2 neutropenia (63.9\%); fatigue (62.3\%); upset stomach (60.0\%); and rash (59.2\%) (q. 27). These commonly encountered toxicities were usually handled by temporary TKI interruption or dose reduction (q. 29).

Table 2 illustrates which toxicities prompted the greatest number of responses indicating drug interruptions, reductions, discontinuations, and switching (q. 29). If pleural effusion, heart failure, or pericardial effusion/pericarditis occurred, approximately $20 \%$ of respondents indicated that they would change to another TKI and approximately $25 \%$ would discontinue therapy.

When asked about events most likely to result in a modification of therapy, 72\% noted grade 3-4 hematological adverse events, $62.7 \%$ grade 3-4 nonhematological adverse events, and $58 \%$ loss of therapeutic response (q. 38). Respondents were asked if they had switched patients with suboptimal response to initial imatinib back to a higher dose or the same dose of imatinib after adverse events or decreased tolerability after being changed to nilotinib or dasatinib, choosing all responses that applied (q. 37). Approximately 50\% of respondents reported never switching patients back to a higher dose or the same dose of imatinib because of adverse events or decreased tolerability to nilotinib or dasatinib; $26 \%$ of the respondents 
reported rarely switching back to imatinib after dasatinib and $18.3 \%$ after nilotinib; and $10.7 \%$ switched several times after dasatinib and $9.9 \%$ after nilotinib.

\section{Discussion}

Clinical practice is rapidly changing because of advances in disease monitoring and more effective treatment modalities. Guideline updates are released annually by NCCN and approximately every 3 years by ELN. Therefore, it is important to understand how physicians are incorporating these recommendations into practice and formulating their preferences as new treatments and management strategies emerge.

The guidelines in place at the time of this survey were the 2010 NCCN guidelines ${ }^{6}$ and 2009 ELN recommendations. ${ }^{5}$ In both guidelines, the overall response to imatinib is classified based on the degree and timing of hematologic, cytogenetic, and molecular responses. The ELN recommendations classify response to first-line imatinib as optimal, suboptimal, or failure. While the $2010 \mathrm{NCCN}$ guidelines ${ }^{6}$ do not specifically address suboptimal response, they developed criteria for continuing treatment or changing therapy because of treatment failure.

In general, the physicians surveyed have practice patterns similar to published guidelines. One notable divergence from the guidelines was in regard to the primary goal for treatment response. According to the $2010 \mathrm{NCCN}$ guidelines, the goal of CML therapy is to achieve CCyR. ${ }^{6}$ However, of the respondents surveyed, only $17.4 \%$ indicated their primary goal for treatment response was CCyR whereas nearly three-quarters (72\%) indicated some level of molecular response.

Achievement of CCyR is a widely accepted goal for CML therapy because cytogenetic responses have been shown to be a significant predictor for survival. ${ }^{6-10}$ Molecular monitoring is markedly more sensitive than conventional cytogenetics and is able to routinely detect much lower levels of disease. ${ }^{11-13}$ Molecular response has been found to be predictive of the duration and loss of CCyR, progression-free survival (PFS), and event-free survival (EFS). ${ }^{7}, 14-22$ Based on the German CML study IV (a prospective, multicenter trial), ${ }^{23}$ MMR at 12 months was associated with significantly better PFS and overall survival (OS) at 3 years compared with no MMR (although an overall survival difference was not observed among patients in CCyR by whether they are in MMR or not). Several studies suggested MMR might not forecast OS despite its predictive effects on PFS and EFS. ${ }^{9,19,24}$ This lack of association might be the result of CCyR loss, triggering a change in therapy. ${ }^{25}$ Thus, additional studies are needed to understand the prognostic value of molecular monitoring on OS.

The NCCN and the ELN propose different end points to define treatment failure or suboptimal response after imatinib, and thus recommend alternate approaches; at 3 months, if less than complete hematologic response (NCCN, ELN) or no cytogenetic response (ELN); 6 months if less than PCyR or no cytogenetic response (NCCN, ELN); 12 months PCyR or less (NCCN, ELN); and 18 months if less than CCyR (NCCN, ELN) or MMR (ELN). According to this survey, respondents were more likely to consider changing therapy if there was no hematologic response at 3 months or no cytogenetic response at 6 months when evaluating suboptimal response to initial TKI therapy. However, the prognostic effect of suboptimal response on PFS is still unclear, and it appears to depend on the timing of suboptimal response..$^{26,27}$

A major advance in CML practice in the time period between this survey (2010) and the previous survey (2005) was approval and availability of nilotinib and dasatinib for first-line and second-line management of CML. Physicians' preference for imatinib $400 \mathrm{mg}$ as the 
initial treatment option decreased from $62 \%$ in 2005 to $52 \%$ in the present survey. In addition, given the options of nilotinib or dasatinib as responses, nearly $40 \%$ of physicians chose them as first-line therapy in the present survey, despite NCCN and ELN recommending imatinib as initial therapy for a newly diagnosed CML patient at the time of this survey.

The survey case question for monitoring initial response to imatinib therapy provides a direct comparison to a question asked in the previous survey (q. 23). Respondents in 2005 most commonly reported using blood counts, cytogenetics, FISH, and qRT-PCR (17\%) followed by blood counts and qRT-PCR (12\%), and blood counts alone (11\%). The most common response to the current survey were peripheral blood counts alone (21.9\%) which likely represents greater confidence in the antileukemic efficacy of TKIs and a reliance on hematologic response and recovery early in treatment.

In the case of the patient with a matched related donor in whom initial imatinib therapy had failed, the response options between the 2005 and 2010 survey differed only in that the imatinib $800 \mathrm{mg}$ option was added. Sixty-nine percent of respondents in 2010 would change therapy to nilotinib or dasatinib in contrast to approximately $50 \%$ choosing nilotinib or dasatinib in 2005. As the 2005 report discussed, nilotinib and dasatinib had not been approved in the United States and there was a question as to how many of the respondents had access to them at that time. Additionally in $2005,26 \%$ of respondents would have recommended immediate allo-SCT and only $10 \%$ would make the same recommendation today. The use of medication combinations including interferon, low dose cytarabine, or hydroxyurea decreased to $1 \%$ in 2010 from $13 \%$ in 2005 . The decrease in the number of respondents recommending allo-SCT in the case of the unrelated matched donor appears proportional to the increased number choosing nilotinib or dasatinib as the next therapeutic option.

Mutations within the BCR-ABL kinase domain are of great concern as they are the most common cause of secondary imatinib resistance and are detected in approximately $50 \%$ of such patients. ${ }^{28-32}$ In comparison with the 2005 survey in which $60 \%$ of respondents indicated that they were unfamiliar with or had never ordered the test for BCR-ABL kinase domain mutations, in 2010 more than half (53.8\%) would test for BCR-ABL kinase domain mutations in patients not achieving MMR 2 years after the initiation of TKI therapy. The number of respondents not familiar with the test, never ordering the test, or not having access to the test decreased to $9 \%$ in the current survey. This demonstrates an increased awareness of the testing for BCR-ABL kinase domain mutations among practitioners over the past 5 years. Understanding a patient's BCR-ABL kinase domain mutation status is vital to determining the next therapeutic strategy: increasing imatinib dose, switching to nilotinib/ dasatinib, moving to allo-SCT, or moving to investigational therapy.

The use of qRT-PCR to measure levels of BCR-ABL has advanced our ability to more accurately measure minimal residual disease. ${ }^{33}$ As with many assays, not all laboratories use standardized methods, so Hughes and colleagues ${ }^{34}$ led a collaboration to create a conversion factor to facilitate all results being reported on a standardized scale. A concerning result of the survey is the degree of unfamiliarity with the International Scale (IS) for BCR-ABL qRT-PCR reporting despite both NCCN and ELN guidelines incorporating the use of the IS for MMR determination. Forty-seven percent of respondents were either unsure or not familiar with the IS. Only $45.6 \%$ responded that they receive qRT-PCR results according to the IS.

Interpretation of the differences between the 2005 and 2010 surveys should be done with caution as the same survey respondents were not used. Additionally, the 2010 survey was 
only conducted with US physicians, whereas the 2005 survey was done with US and European physicians (76\% and 24\%, respectively).

The responses are based on physicians' opinions and theoretic scenarios; therefore, they may not be reflective of prescription trends as the accessibility of medications might be constrained for practitioners in their actual practice yet not in the survey (eg, formulary restrictions, insurance coverage). In survey research there is the possibility of question ambiguity (eg, no clear definition or defined time period) which may have led to interpretation of questions differently and responding based on individual respondents' interpretation. Other limitations include potential lack of availability of certain monitoring tests (eg, qRT-PCR, FISH) for all respondents and the allowance for multiple answers in selected questions. Allowing for multiple responses on individual questions can make interpretation and comparisons difficult; however it does provide the ability to gain a comprehensive answer from respondents.

\section{Conclusion}

Despite clear evidence from clinical trials and guidelines, this survey demonstrates a possible shift of practice with treatment goals to achieve MMR when CCyR has been achieved. Additionally, nilotinib and dasatinib were noted as being used increasingly for first-line treatment though guidelines at the time supported initial treatment with imatinib. Nilotinib and dasatinib had only recently been approved for first-line treatment of CML at the time of this survey; therefore the effect of these agents on practice changes might be underestimated in this survey.

Since the time of this survey, CML management has continued to evolve rapidly. The 2011 and 2012 NCCN guidelines ${ }^{4,35}$ include nilotinib and dasatinib as first-line treatment options and monitoring practices and definitions continue to be explored. The results of this survey and ongoing advances in CML management illustrate the need for additional clinical trials and real world data as well as education regarding current guideline recommendations and their implementation in the treatment and monitoring of patients with CML.

\section{Supplementary Material}

Refer to Web version on PubMed Central for supplementary material.

\section{Acknowledgments}

This study was funded by Novartis Pharmaceuticals Corporation. The authors thank Peter Herout, PharmD, for his assistance in developing the interview questions, project management, and summary of findings for this study.

Medefield was the survey company that recruited study participants, conducted the online survey, and provided administrative services (processing honoraria, building the online survey, providing technical support, etc).

\section{References}

1. Kantarjian HM, Cortes J, Guilhot F, et al. Diagnosis and management of chronic myeloid leukemia: a survey of American and European practice patterns. Cancer. 2007; 109:1365-75. [PubMed: 17326047]

2. Saglio G, Kim DW, Issaragrisil S, et al. Nilotinib versus imatinib for newly diagnosed chronic myeloid leukemia. N Engl J Med. 2010; 362:2251-9. [PubMed: 20525993]

3. Kantarjian H, Shah NP, Hochhaus A, et al. Dasatinib versus imatinib in newly diagnosed chronicphase chronic myeloid leukemia. N Engl J Med. 2010; 362:2260-70. [PubMed: 20525995] 
4. National Comprehensive Cancer Network. [Accessed February 1, 2012] NCCN: Clinical Practice Guidelines in Oncology, Chronic Myelogenous Leukemia Version 2. 2012. Available at: http:// www.ncen.org/professionals/physician_gls/f_guidelines.asp

5. Baccarani M, Cortes J, Pane F, et al. Chronic myeloid leukemia: an update of concepts and management recommendations of European LeukemiaNet. J Clin Oncol. 2009; 27:6041-51. [PubMed: 19884523]

6. National Comprehensive Cancer Network. [Accessed January 31, 2010] NCCN: Clinical Practice Guidelines in Oncology, Chronic Myelogenous Leukemia Version 1. 2010. Available at: http:// www.nccn.org/professionals/physician_gls/f_guidelines.asp

7. Druker BJ, Guilhot F, O'Brien SG, et al. Five-year follow-up of patients receiving imatinib for chronic myeloid leukemia. N Engl J Med. 2006; 355:2408-17. [PubMed: 17151364]

8. Deininger M, O'Brien SG, Guilhot F, et al. International randomized study of interferon vs STI571 (IRIS) 8-year follow up: sustained survival and low risk for progression or events in patients with newly diagnosed chronic myeloid leukemia in chronic phase (CML-CP) treated with imatinib. Blood (ASH Annual Meeting Abstracts). 2009; 114:462. [ASH Abstract 1126].

9. de Lavallade H, Apperley JF, Khorashad JS, et al. Imatinib for newly diagnosed patients with chronic myeloid leukemia: incidence of sustained responses in an intention-to-treat analysis. J Clin Oncol. 2008; 26:3358-63. [PubMed: 18519952]

10. Quintás-Cardama A, Cortes JE, O'Brien S, et al. Dasatinib early intervention after cytogenetic or hematologic resistance to imatinib in patients with chronic myeloid leukemia. Cancer. 2009; 115:2912-21. [PubMed: 19402171]

11. Branford S, Hughes TP, Rudzki Z. Monitoring chronic myeloid leukaemia therapy by real-time quantitative PCR in blood is a reliable alternative to bone marrow cytogenetics. Br J Haematol. 1999; 107:587-99. [PubMed: 10583264]

12. Schoch C, Schnittger S, Bursch S, et al. Comparison of chromosome banding analysis, interphaseand hypermetaphase-FISH, qualitative and quantitative PCR for diagnosis and for follow-up in chronic myeloid leukemia: a study on 350 cases. Leukemia. 2002; 16:53-9. [PubMed: 11840263]

13. Jabbour EJ, Quintás-Cardama A. Molecular monitoring 101: helping your patients with CML understanding the meaning of molecular response. Leuk Lymphoma. 2012; 53:1452-60. [PubMed: 22273251]

14. Hughes TP, Hochhaus A, Branford S, et al. Long-term prognostic significance of early molecular response to imatinib in newly diagnosed chronic myeloid leukemia: an analysis from the international randomized study of interferon and STI571 (IRIS). Blood. 2010; 116:3758-65. [PubMed: 20679528]

15. Cortes J, Talpaz M, O'Brien S, et al. Molecular responses in patients with chronic myelogenous leukemia in chronic phase treated with imatinib mesylate. Clin Cancer Res. 2005; 11:3425-32. [PubMed: 15867244]

16. Iacobucci I, Saglio G, Rosti G, et al. Achieving a major molecular response at the time of a complete cytogenetic response (CCgR) predicts a better duration of $\mathrm{CCgR}$ in imatinib-treated chronic myeloid leukemia patients. Clin Cancer Res. 2006; 12:3037-42. [PubMed: 16707599]

17. Palandri F, Iacobucci I, Soverini S, et al. Treatment of Philadelphia-positive chronic myeloid leukemia with imatinib: importance of a stable molecular response. Clin Cancer Res. 2009; 15:1059-63. [PubMed: 19188180]

18. Paschka P, Müller MC, Merx K, et al. Molecular monitoring of response to imatinib (Glivec) in CML patients pretreated with interferon alpha. Low levels of residual disease are associated with continuous remission. Leukemia. 2003; 17:1687-94. [PubMed: 12970765]

19. Marin D, Milojkovic D, Olavarria E, et al. European LeukemiaNet criteria for failure or suboptimal response reliably identify patients with CML in early chronic phase treated with imatinib whose eventual outcome is poor. Blood. 2008; 112:4437-44. [PubMed: 18716134]

20. Press RD, Galderisi C, Yang R, et al. A half-log increase in bcr-abl RNA predicts a higher risk of relapse in patients with chronic myeloid leukemia with an imatinib-induced complete cytogenetic response. Clin Cancer Res. 2007; 13:6136-43. [PubMed: 17947479] 
21. Press RD, Love Z, Tronnes AA, et al. BCR-ABL mRNA levels at and after the time of a complete cytogenetic response (CCR) predict the duration of CCR in imatinib mesylate-treated patients with CML. Blood. 2006; 107:4250-6. [PubMed: 16467199]

22. Hughes TP, Kaeda J, Branford S, et al. Frequency of major molecular responses to imatinib or interferon alfa plus cytarabine in newly diagnosed chronic myeloid leukemia. N Engl J Med. 2003; 349:1423-32. [PubMed: 14534335]

23. Hehlmann R, Lauseker M, Jung-Munkwitz S, et al. Tolerability-adapted imatinib $800 \mathrm{mg} / \mathrm{d}$ versus $400 \mathrm{mg} / \mathrm{d}$ versus $400 \mathrm{mg} / \mathrm{d}$ plus interferon- $a$ in newly diagnosed chronic myeloid leukemia. J Clin Oncol. 2011; 29:1634-42. [PubMed: 21422420]

24. Kantarjian H, O'Brien S, Shan J, et al. Cytogenetic and molecular responses and outcome in chronic myelogenous leukemia: need for new response definitions? Cancer. 2008; 112:837-45. [PubMed: 18085610]

25. Cortes J, Quintás-Cardama A, Kantarjian HM. Monitoring molecular response in chronic myeloid leukemia. Cancer. 2011; 117:1113-22. [PubMed: 20960522]

26. Alvarado Y, Kantarjian H, O'Brien S, et al. Significance of suboptimal response to imatinib, as defined by the European LeukemiaNet, in the long-term outcome of patients with early chronic myeloid leukemia in chronic phase. Cancer. 2009; 115:3709-18. [PubMed: 19517462]

27. Marin D, Milojkovic D, Olavarria E, et al. European LeukemiaNet criteria for failure or suboptimal response reliably identify patients with CML in early chronic phase treated with imatinib whose eventual outcome is poor. Blood. 2008; 112:4437-44. [PubMed: 18716134]

28. Branford S, Hughes T. Detection of BCR-ABL mutations and resistance to imatinib mesylate. Methods Mol Med. 2006; 125:93-106. [PubMed: 16502579]

29. Hochhaus A, Kreil S, Corbin AS, et al. Molecular and chromosomal mechanisms of resistance to imatinib (STI571) therapy. Leukemia. 2002; 16:2190-6. [PubMed: 12399961]

30. Jabbour E, Kantarjian H, Jones D, et al. Frequency and clinical significance of BCR-ABL mutations in patients with chronic myeloid leukemia treated with imatinib mesylate. Leukemia. 2006; 20:1767-73. [PubMed: 16855631]

31. Lahaye T, Riehm B, Berger U, et al. Response and resistance in 300 patients with BCR-ABLpositive leukemias treated with imatinib in a single center: a 4. 5-year follow-up. Cancer. 2005; 103:1659-69. [PubMed: 15747376]

32. Soverini S, Colarossi S, Gnani A, et al. Contribution of Abl kinase domain mutations to imatinib resistance in different subsets of Philadelphia-positive patients: by the GIMEMA Working Party on Chronic Myeloid Leukemia. Clin Cancer Res. 2006; 12:7374-9. [PubMed: 17189410]

33. Branford S. Chronic myeloid leukemia: molecular monitoring in clinical practice. Hematology Am Soc Hematol Educ Program. 2007:376-83. [PubMed: 18024654]

34. Hughes T, Deininger M, Hochhaus A, et al. Monitoring CML patients responding to treatment with tyrosine kinase inhibitors: review and recommendations for harmonizing current methodology for detecting BCR-ABL transcripts and kinase domain mutations and for expressing results. Blood. 2006; 108:28-37. [PubMed: 16522812]

35. National Comprehensive Cancer Network. [Accessed March 31, 2011] NCCN: Clinical Practice Guidelines in Oncology, Chronic Myelogenous Leukemia. Version 2. 2011. http://www.nccn.org/ professionals/physician_gls/f_guidelines.asp 


\section{Clinical Practice Points}

- In 2005, a survey was conducted evaluating physician-reported practice patterns in the management of CML.

- The NCCN publishes new guidelines yearly for CML and ELN most recently updated their recommendations in 2009.

- The 2010 NCCN guidelines and 2009 ELN recommendations added nilotinib and dasatinib to their treatment algorithms.

- The current survey reevaluated the self-reported management of CML among board certified hematologists/oncologists considering the many changes in CML treatment options and management strategies in the past 5 years.

- Imatinib $400 \mathrm{mg}$ as the initial treatment option has decreased from $62 \%$ in 2005 to $52 \%$ in the 2010 survey. Now nearly $40 \%$ of physicians would choose nilotinib/dasatinib as first-line treatment.

- From the surveyed physicians, achievement of at least MMR is the predominant treatment goal in chronic phase CML, and most would adjust the imatinib dose or change therapy to nilotinib/dasatinib in order to achieve MMR even after CCyR is achieved.

- This survey demonstrates decisions are influenced not only by guidelines but on the attitudes, beliefs, and personal experience of the responding physicians.

- These observations provide insights on current treatment practices, the importance for alternative approaches to education than just guideline dissemination, and need for further clinical trials and real world data on the prognostic value of MMR. 


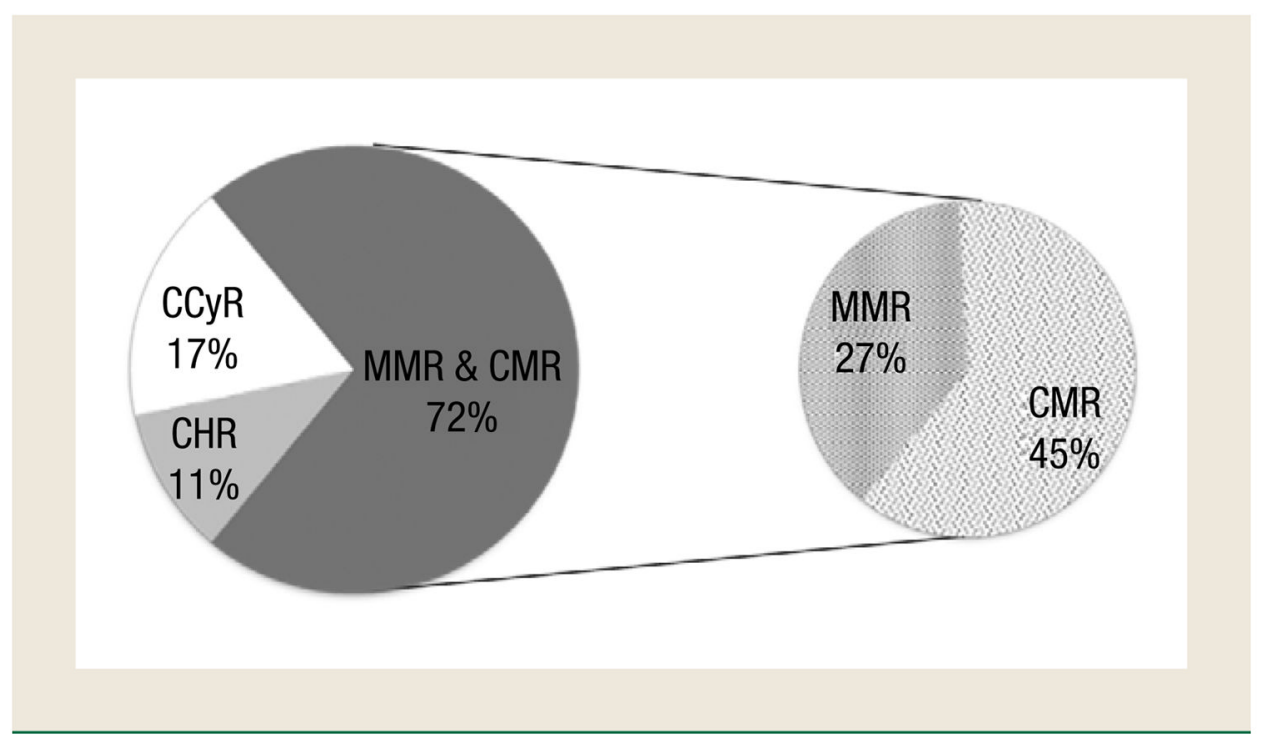

Figure 1. Survey Respondents' Primary Goal for Treatment Response in a Newly Diagnosed Chronic Myeloid Leukemia Patient Being Treated With a Tyrosine Kinase Inhibitor (Question 13)

Abbreviations: $\mathrm{CCyR}=$ complete cytogenetic response; $\mathrm{CHR}=$ complete hematologic response; $\mathrm{CMR}=$ complete molecular response; $\mathrm{MMR}=$ major molecular response. 


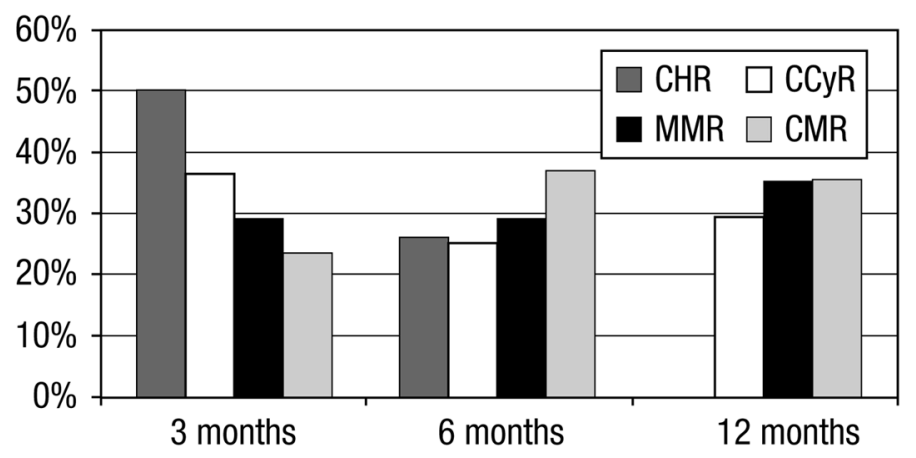

Figure 2. Survey Respondents' Most Critical Time Point to Evaluate Response to First-Line Tyrosine Kinase Inhibitor Therapy, Based on the Question From Figure 1 (Question 14) Abbreviations: $\mathrm{CCyR}=$ complete cytogenetic response; $\mathrm{CHR}=$ complete hematologic response; $\mathrm{CMR}=$ complete molecular response; $\mathrm{MMR}=$ major molecular response. 


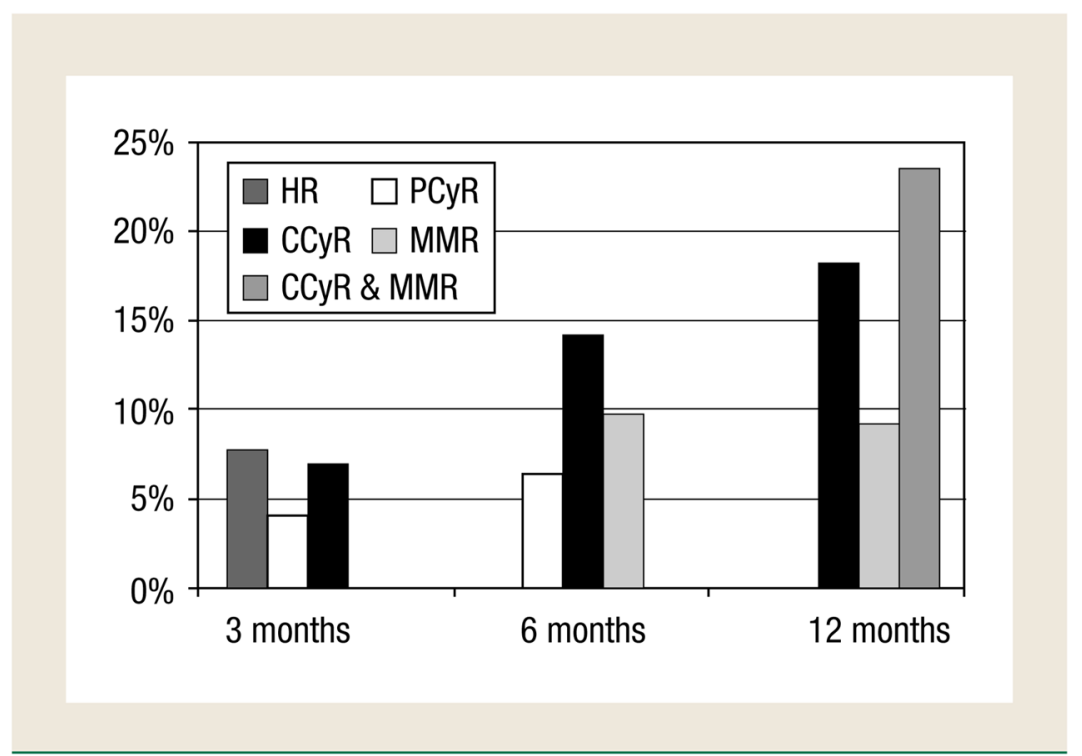

Figure 3. Survey Respondents' Assessment of Effective Tyrosine Kinase Inhibitor Therapy When Treating Newly Diagnosed Chronic Phase Chronic Myeloid Leukemia Patients (Question 25)

Abbreviations: $\mathrm{CCyR}=$ complete cytogenetic response; $\mathrm{HR}=$ hematologic response; $\mathrm{MMR}$ = major molecular response $\mathrm{PCyR}=$ partial cytogenetic response. 


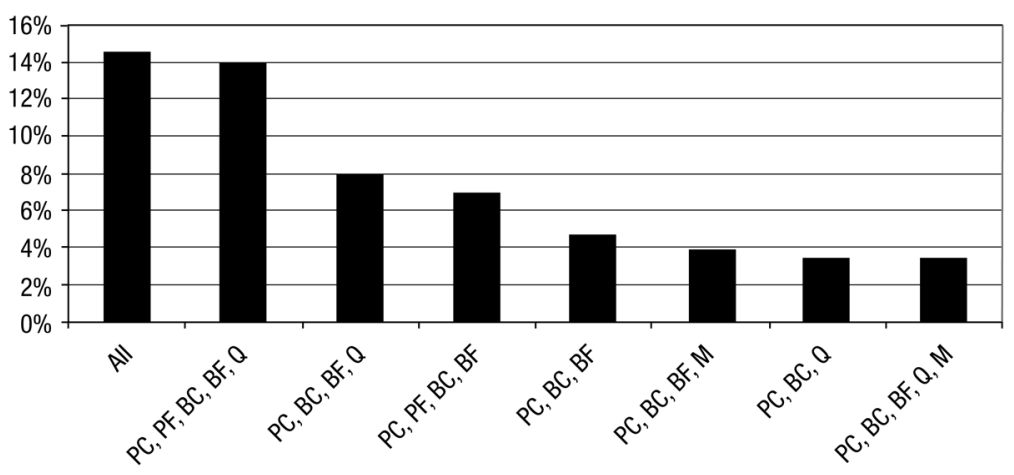

Figure 4. Combination of Tests Used to Monitor Initial Response to Oral Tyrosine Kinase Inhibitor Therapy (Question 15). Multiple Answers were Allowed

Abbreviations: $\mathrm{BC}=$ bone marrow cytogenetics; $\mathrm{BF}=$ bone marrow FISH; FISH = fluorescence in situ hybridization; $\mathrm{M}=$ breakpoint cluster region-abelson (BCR-ABL) kinase domain mutation analysis; $\mathrm{PC}=$ peripheral blood cell count; $\mathrm{PF}=$ peripheral blood FISH; Q = quantitative reverse-transcriptase polymerase chain reaction. 
Table 1

Survey Results of Determination of Suboptimal Response to Therapy (Questions 33 and 24) ${ }^{a}$

\begin{tabular}{|l|c|c|}
\hline Response Option $^{\boldsymbol{a}}$ & General, $\mathbf{n}(\boldsymbol{\%})$ & Case Based, $\mathbf{n}(\boldsymbol{\%})$ \\
\hline No HR at 30 Months & $223(44)$ & $276(54.4)$ \\
\hline Less Than CHR at 3 Months & $155(30.6)$ & $181(35.7)$ \\
\hline Less Than CHR at 6 Months & $204(40.2)$ & $215(42.4)$ \\
\hline No Cytogenetic Response at 6 Months & $217(42.8)$ & $256(50.5)$ \\
\hline Less Than PCyR at 6 Months & $143(28.2)$ & $178(35.1)$ \\
\hline Less Than PCyR at 12 Months & $165(32.5)$ & $194(38.3)$ \\
\hline Less Than CCyR at 12 Months & $183(36.1)$ & $183(36.1)$ \\
\hline Less Than CCyR at 18 Months & $132(26.0)$ & $160(31.6)$ \\
\hline Less Than MMR at 18 Months & $192(37.9)$ & $192(37.9)$ \\
\hline Loss of CHR or CCyR Any Time & $201(39.6)$ & $206(40.6)$ \\
\hline Loss of MMR Any Time & $158(31.2)$ & $148(29.2)$ \\
\hline
\end{tabular}

Abbreviations: $\mathrm{CCyR}$, complete cytogenetic response; $\mathrm{CHR}=$ complete hematologic response; $\mathrm{HR}=$ hematologic remission; $\mathrm{MMR}$, major molecular response; PCyR, partial cytogenetic response.

a Multiple answers were allowed. 
Table 2

Severe Toxicities Causing Physicians to Interrupt, Dose Reduce, Discontinue, or Switch a Patient's Tyrosine Kinase Inhibitor Therapy (Question 29) ${ }^{a}$

\begin{tabular}{|l|c|c|c|c|}
\hline Toxicity & \% Interrupt & \% Dose Reduce & \% Discontinue & \% Switch \\
\hline Nausea/Vomiting & 30.8 & 34.3 & 5.1 & 9.1 \\
\hline Upset Stomach & 20.1 & 29.8 & 3.0 & 5.9 \\
\hline Diarrhea & 25.2 & 36.3 & 5.1 & 8.3 \\
\hline Rash & 26.2 & 29.2 & 6.7 & 10.5 \\
\hline Neutropenia & 32.7 & 47.7 & 7.3 & 8.9 \\
\hline Thrombocytopenia & 29.0 & 45.4 & 8.5 & 8.3 \\
\hline Anemia & 19.5 & 36.9 & 4.7 & 4.5 \\
\hline Muscle Cramps & 20.7 & 27.6 & 3.2 & 6.9 \\
\hline Liver Dysfunction & 22.9 & 31.4 & 15.4 & 16.6 \\
\hline Fluid Retention & 20.1 & 33.9 & 7.9 & 14.0 \\
\hline Periorbital Edema & 17.6 & 27.8 & 5.1 & 8.3 \\
\hline Pleural Effusion & 22.7 & 24.5 & 21.7 & 27.6 \\
\hline Pericardial Effusion or Pericarditis & 17.0 & 16.6 & 19.1 & 24.9 \\
\hline Weight Gain & 16.0 & 23.1 & 3.4 & 6.9 \\
\hline Congestive Heart Failure & 15.4 & 16.0 & 21.1 & 26.4 \\
\hline Fatigue & 19.1 & 33.5 & 3.2 & 9.7 \\
\hline Bone Aches & 17.0 & 25.8 & 2.8 & 5.7 \\
\hline
\end{tabular}

${ }^{a}$ Multiple answers were allowed. 